\title{
Research on Passenger Flow Assignment of High-Speed Trains Based on Personalized Itinerary Choice
}

\author{
Jinzi Zheng (iD) \\ Railway Big Data Research and Application Innovation Center, China Academy of Railway Sciences Corporation Limited, \\ No. 2 Daliushu Road, Haidian District, Beijing 100081, China
}

Correspondence should be addressed to Jinzi Zheng; 10114214@bjtu.edu.cn

Received 20 January 2020; Revised 28 March 2020; Accepted 1 April 2020; Published 1 May 2020

Academic Editor: Ewa Pawluszewicz

Copyright ( 2020 Jinzi Zheng. This is an open access article distributed under the Creative Commons Attribution License, which permits unrestricted use, distribution, and reproduction in any medium, provided the original work is properly cited.

Under the condition that ticket fare for high-speed train could fluctuate around a benchmark price in China, aimed at predicting how the passengers will distribute among different trains given a ticket fare, the passenger flow assignment method for high-speed trains is studied. Different from the classical researches on the passenger flow assignment, by introducing a variable that represents the value of time, this research allows passengers to make their personalized choice between the principles of time minimization and expense minimization, so as to demonstrate how the passengers holding different time values respond to each ticket fare scheme. An equilibrium passenger flow assignment model based on personalized choice is built and an improved Monte-Carlo random simulation algorithm is designed for solving the model. The actual ticket sale data for Beijing-Shanghai high-speed railway are used to verify the feasibility of the proposed model and algorithm. The passenger flow assignment results under various fare schemes show how the distribution of passenger flow changes with the adjustment of ticket fare.

\section{Introduction}

Although China's high-speed train has achieved tremendous results during the last decade, passenger flow is generally unevenly distributed between trains due to different departure and arrival time, travel time, and stopping schedule. The published prices of high-speed train tickets may be properly adjusted based on actual demands to effectively regulate passenger flow distribution among trains, enhance resource utilization, and increase transport enterprises' revenues. However, passengers' itinerary choice will surely be affected by the adjustment of ticket fare. After the continual iteration and feedback between ticket fare adjustment and passengers' itinerary choice, an optimal fare scheme is eventually formulated to meet the needs of both transport enterprises and passengers. Therefore, passenger flow assignment at a certain ticket fare is a key link in adjusting and optimizing ticket fare.

On the basis of the theory and method of traffic flow distribution for urban roads $[1,2]$, China's scholars studied passenger flow assignment for railway network, aiming to evaluate and optimize operation schemes [3-5]. In some researches, it is a spatial transfer network based on operation schemes. Service network design is one of research hotspots in passenger flow assignment. A variety of transfer networks are introduced in literature [6], in which their scales, functions, and computing capacities are compared and analyzed. Based on problems, this paper introduces a threedimensional time-space service network $[7,8]$, constructs passenger flow assignment network based on train schedules and itinerary choice, and proposes a way of calculating path impedance while considering passengers' preferences on departure time.

In researches about traditional traffic distribution, it is assumed that all passengers select their preferred paths based on minimum travel time and lowest equivalent expense. However, in our daily life, passengers' preferences to time and expense may differ mainly for their different income levels and trip purposes. High-income business travelers focus more on time efficiency, while workers and leisure passengers are more sensitive to expense. To truly demonstrate passengers' responses to ticket strategy, they should be allowed to have heterogeneous trade-offs between 
minimum time and lowest fare. The multiclass passenger flow assignment [9-11] is more practical as population diversity has been taken into account. A time-fare bi-criteria equilibrium passenger flow assignment model was proposed in this paper based on passengers' personalized itinerary choice and transport features, and an improved MonteCarlo random simulation algorithm was designed to solve the model. And the actual data of Beijing-Shanghai highspeed railway were also introduced to verify the proposed method, based on which how passenger flow distribution changes with the adjustment of ticket fare is finally discussed.

\section{Model Assumptions and Network Construction}

In this section, we first construct a service network representing service paths of high-speed railway. After that, the definition of major notations and the travel cost calculation method are clarified.

Consider a high-speed railway network with 4 stations and railway lines between them. Each station is represented as a node, that is, A, B, C, and D, respectively (see Figure 1). There are three trains, represented as T1, T2, and T3, running on this network in the direction of $\mathrm{A}$ to $\mathrm{D}$. As shown in the train running diagram (see Figure 2), T1 runs from A to $\mathrm{D}$ and stops at $\mathrm{B}$ and $\mathrm{C}, \mathrm{T} 2$ runs from $\mathrm{B}$ to $\mathrm{D}$ and stops at $\mathrm{C}$, and $\mathrm{T} 3$ runs from $\mathrm{A}$ to $\mathrm{D}$ and stops at $\mathrm{C}$.

A trip in this study begins when a passenger gets aboard at the departure station and ends when he or she gets off at the arrival station. Based on the above physical network and train running diagram, a three-dimensional time-space network is constructed to depict service paths for travelling, as shown in Figure 3. In this service network, one-dimensional coordinate represents time, while other two-dimensional coordinates represent train running direction and passenger embarkation direction, respectively. The timespace service network in Figure 3 is expressed by $G=(N, E)$, in which $N$ is the set of nodes. There are six node types in the service network, which are the boarding node at departure station, the getting-off node at arrival station, the traindeparture node, the train-arrival node, the arrival node at intermediate station, and the departure node at intermediate station. $E$ is the set of segments. There are five segment types in the service network, which are the operation arc, the stop arc, the boarding arc at departure station, the getting-off arc at arrival station, and the transfer arc. The trip path between the boarding node at departure station and the getting-off node at arrival station is defined as a service path, which can be expressed by the node sequence in this path. Take the node pair A and D for example, between them there exists a direct service path $r_{1}^{\mathrm{AD}}$ expressed as the nodes sequence 1-23-5-7-9-11-12; there also exists a transit service path $r_{2}^{\mathrm{AD}}$ expressed as the nodes sequence 1-2-3-14-15-17-19-20. To better depict passenger's trip in the model, the set of train and any segment that the train passes through is built, which is expressed as (train-segment). For example, the set of T1 and its segment $A B$ could be expressed as (T1-AB). All the

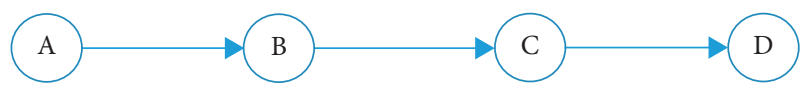

FIGURE 1: Railway physical network.

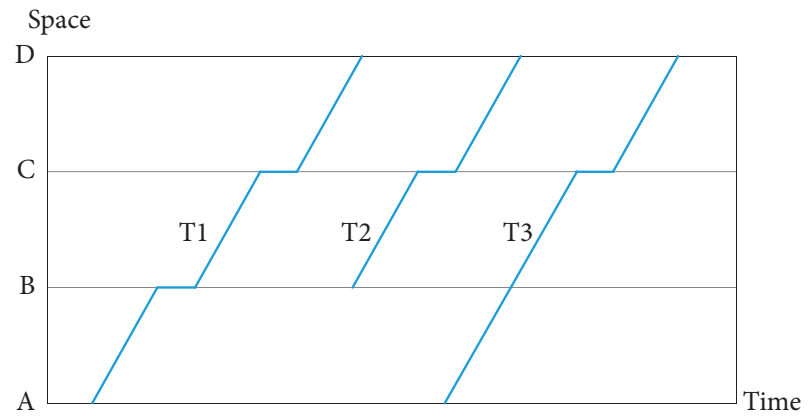

FIGURE 2: Train running diagram.

sets of train-segment included in service path $r$ are represented by $S_{r}$.

Based on the service network as indicated in Figure 3, major notations are defined as follows:

$W=\{w\}$ : set of passenger flow $\mathrm{OD}$

$G=(N, E)$ : service network, in which $N$ is the set of service node and $E$ is the set of segment

$n$ : service node in the train service network, $n \in N$

$e$ : segment in the train service network, $e \in E$

$R=\{r\}$ : set of all service paths in the train service network

$R_{w}=\left\{r_{w}\right\}:$ set of OD in $w$ service path, $w \in W$

$S=\{s\}$ : set of all (train-segment)

$p_{r}$ : ticket fare of service path $r, r \in R$

$g_{w}$ : total demand for $w$ by OD, $w \in W$

$x_{r_{w}}$ : number of passengers selecting service path $r$ in $w$ by $\mathrm{OD}$, or passenger flow, $r_{w} \in R_{w}, w \in W$

$x_{e}$ : passenger flow in segment $e, e \in E$

$t_{e}$ : running duration in segment $e, e \in E$

$h_{e}$ : maximum passengers for segment, $e \in E$

$\delta_{e r}$ : segment-service path occupation index. If service path $r$ occupies segment $e, \delta_{e r}=1$, otherwise, $\delta_{e r}=0$, $e \in E$ and $r \in R$

$f(\alpha)$ : probability density function of passengers' value of time

Q: passengers' average on-boarding and off-boarding speed, unit is people/min

The factors affecting passengers' railway option include travel time, expense, and departure time. Thus, the generalized expense of service path $r$ is

$$
C^{r}=\beta_{T} C_{T}^{r}+\beta_{P} C_{P}^{r}+\beta_{d e} D_{d e}^{r} .
$$

In the equation, $C_{T}^{r}, C_{P}^{r}$, and $D_{d e}^{r}$ represent the costs of travel time, expense, and departure time of a passenger whose departure time is earlier or later, respectively. $\beta_{T}, \beta_{P}$, 


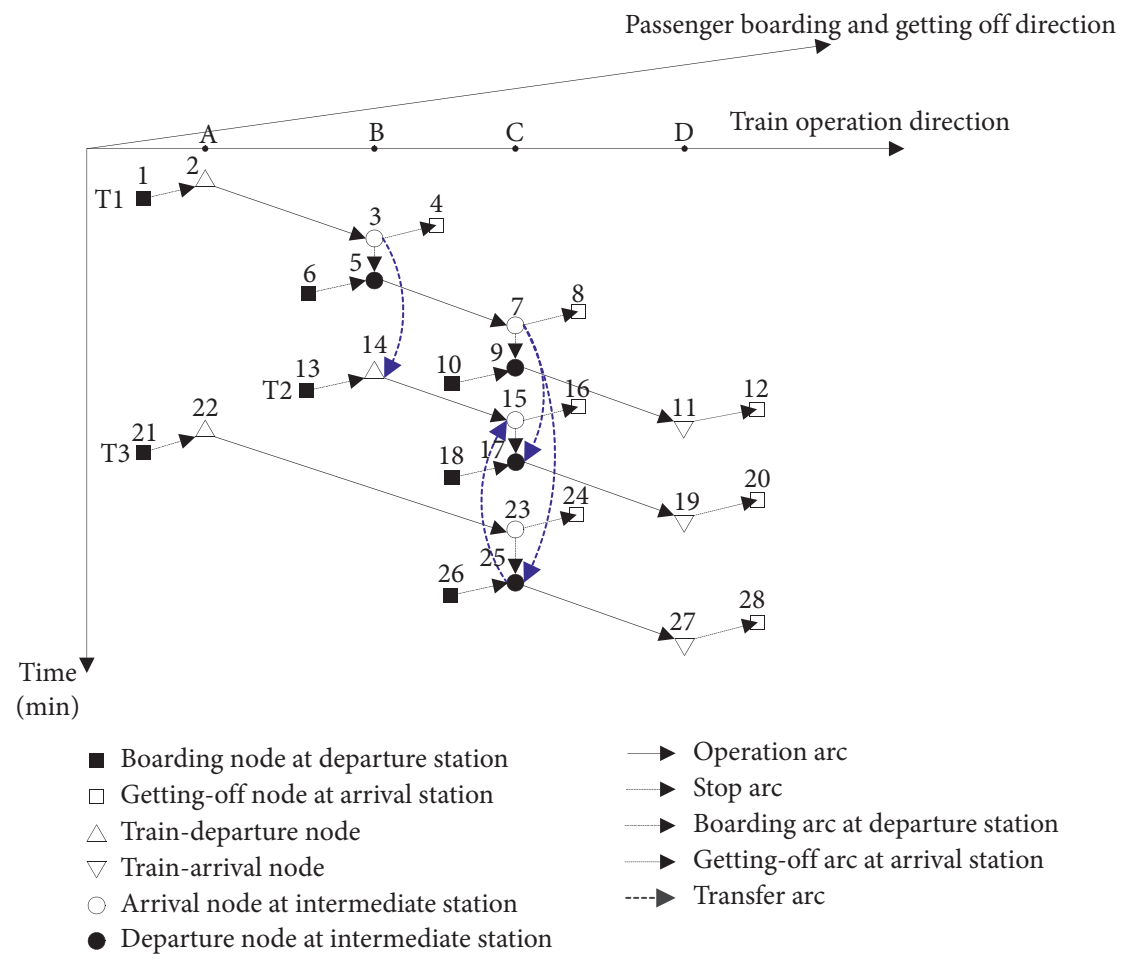

FIgURE 3: Three-dimensional time-space service network.

and $\beta_{d e}$ are the coefficients for calculating each cost, respectively. Based on service network in Figure 1, each cost can be specifically calculated as follows.

2.1. Cost of Travel Time. Cost of travel time reflects the travel time required by the service path, and it is the sum of time of each segment making up the service path. The time of running segment $E_{1}$, stopping segment $E_{2}$, and transfer segment $E_{5}$ is given in train schedules, while that of segments of getting aboard at the departure station $E_{3}$ and getting off at the arrival station $E_{4}$ is the ratio of passenger flow in segments and passengers' average on-boarding and off-boarding speed. Thus, below is how the cost of travel time $C_{T}^{r}$ in service path $r$ can be calculated:

$$
C_{T}^{r}=\sum_{e \in E_{1} \cup E_{2} \cup E_{5}} \delta_{e r} t_{e}+\sum_{e \in E_{3} \cup E_{4}} \frac{\delta_{e r} x_{e}}{Q} .
$$

2.2. Cost of Expense. The expense of the service path is the sum of ticket fares of all train-segment. If a passenger's value of time (expense per unit time) is $\alpha$, then the cost of expense for service path $r$ is

$$
C_{P}^{r}(\alpha)=\frac{\sum_{s \in S_{r}} p_{s}}{\alpha}
$$

Among which, $\alpha$ is a random variable, demonstrating passengers' perceptions about time and expense. The probability density function $f(\alpha)$ indicates how passengers' value of time is distributed.
2.3. Schedule Delay Cost. High-speed trains run according to diagrams, and departure time is exactly arranged; therefore, departure time greatly affects passengers' itinerary choice. The absolute value of the difference between actual and ideal departure time in the service path is used as schedule delay cost to quantitatively express the bother caused to passengers by the difference between actual and ideal departure time. The running duration in one day is divided into $\mathrm{M}$ time slots. The intermediate time $t(m)$ in time slot $m$ is used to approximately represent the ideal departure time of the passengers who wish to departure in this time slot. $F(m)$ represents the probability of passengers' ideal departure time to be within time slot $m$. The schedule delay cost $D_{d e}^{r}(\tau)$ for the service path $r$ with the actual departure time $\tau$ can be calculated as follows:

$$
D_{d e}^{r}(\tau)=\sum_{m=1}^{M} F(m)|t(m)-\tau| .
$$

\section{Construction and Solution of Passenger Flow Assignment Model}

3.1. Equilibrium Passenger Flow Assignment Model. Passengers may accurately estimate the generalized cost of all service paths based on train schedule before trip and make correct decisions based on their own value. If passengers' choice on service path is based on their minimum perceived generalized cost, then the generalized costs of the segments of getting aboard at the departure station and getting off at the arrival station will increase correspondingly as passenger flow in the service path increases till that of the 
service path is minimized. Then, passengers may select the path with newly perceived minimum generalized cost so that passenger flow can be evenly distributed in all service paths, namely, the cost of time for the service path to be selected by the same OD pair is the lowest among the service paths at the same expense, whose passengers, moreover, hold the same value of time [10].

All service paths for OD in segment $w$ are divided into $K_{w}$ categories based on expense. The expense for path $i$ is expressed by $p_{i}^{w}$, and $G_{i}^{w}$ represents the total path flow whose expense is not higher than that of path $i$. The accumulative density function of the value of time is expressed $\operatorname{as} F_{w}(\alpha)=\int_{0}^{\alpha} f(\omega) d \omega$. If $E_{w}(\theta)=\int_{0}^{\theta}\left(1 / F_{w}^{-1}(\omega)\right) d \omega$, then $E_{w}\left(G_{i}^{w} / g_{w}\right)-E_{w}\left(G_{i-1}^{w} / g_{w}\right)$ will express the countdown of passengers' value of time by OD pair in segment $w$ and path $i$. The equilibrium passenger flow assignment flow is thus constructed as follows:

$$
\begin{aligned}
& \min \quad Z=\sum_{e \in E_{3} \cup E_{4}} \int_{0}^{x_{e}} t_{e}(\omega) d \omega+\sum_{w \in W} g_{w}\left\{\sum_{i=1}^{K_{w}} p_{i}^{w}\left[E_{w}\left(\frac{G_{i}^{w}}{g_{w}}\right)-E_{w}\left(\frac{G_{i-1}^{w}}{g_{w}}\right)\right]\right\} \\
& \text { s.t. } \quad x_{e}=\sum_{w \in W} \sum_{r \in R_{w}} \delta_{e r} x_{r}^{w}, \quad e \in E \\
& \sum_{r \in R_{w}} x_{r}^{w}=g_{w}, \quad w \in W \\
& x_{e} \leq h_{e}, \quad e \in E_{1} \\
& x_{r}^{w} \geq 0, w \in W, \quad r \in R_{w} .
\end{aligned}
$$

The first section of (5) is the objective function of classical equilibrium passenger flow assignment problem, and the second section minimizes all passengers' perceived cost of expense. The relationship between the flow of segment and path is shown in (6). Based on (7), the sum of the flow of all paths by the same OD pair equals to OD demand quantity. Equation (8) is the constraint of train capacity to ensure that the flow of any segment is not higher than the capacity of the train that the segment is located in, which is expressed by segment capacity $h_{e}$. Equation (9) is the nonnegative constrain of independent variable.

3.2. Passenger Flow Assignment Algorithm. The model above is not a classical flow distribution model, which could not be solved by the classical algorithms. Stimulated by the solution idea presented by Leurent [10], an improved Monte-Carlo random simulation algorithm is designed according to the characteristics of the model. The algorithm's basic idea is to generate the set of service paths for each OD pair, calculate the generalized cost of each service path based on the value of time at point $\mathrm{O}$ randomly produced in the iteration, and upload OD demand into $\mathrm{K}$ shortest paths according to the remaining capacity of the service paths. OD is randomly selected for passenger flow assignment to prevent its order affecting the result. The following is how the algorithm specifically works:

Step 1. Initialization. Give a sequence of real numbers: $\eta_{k}$, $0 \leq \eta_{k} \leq 1, \quad \sum \eta_{k}=\infty$, and $\sum \eta_{k}^{2}<\infty$. And initialize the remaining capacity of each segment while making $z_{e}^{0}=h_{e}$ and $e \in E_{1}$.
Step 1.1. Randomly select OD pair $w$, list all service paths, calculate the generalized cost of each service path based on the initial generalized cost of each segment (cost of expense is not included), and put the generalized costs of all paths into the set $R_{w}$ from small to large.

Step 1.2. Select the path $r_{w}^{1}$ with the lowest generalized cost from $R_{w}$, check the remaining capacity of the segments that it includes $z_{e}^{n}\left(\delta_{e r^{w}}=1\right)$, and take the minimum remaining capacity as its available capacity $z^{n}\left(r_{w}^{1}\right)$; allocate OD demand of $\min \left\{z^{n}\left(r_{w}^{1}\right), g_{w}\right\}$ into the path $r_{w}^{1}$ and update the remaining capacity of corresponding segment $z_{e}^{n}=z_{e}^{n}-$ $\min \left\{z^{n}\left(r_{w}^{1}\right), g_{w}\right\}$ and OD demand $g_{w}=g_{w}-\min \left\{z^{n}\left(r_{w}^{1}\right)\right.$, $\left.g_{w}\right\}$, if $g_{w}=0$, go to Step 1.3; otherwise, select the secondary path $r_{w}^{2}$ and repeat Step 1.2.

Step 1.3. If some OD pairs are not distributed in the set of $W$, go back to Step 1.1; if all OD demands are distributed, then obtain the initial flow $x_{e}^{0}$ of each segment and make the times of iteration $n=0$; the generalized cost of the segment will be $c_{e}^{0}=c_{e}(0), \forall e \in E$.

Step 2. Update the generalized cost of the segment. Make $n=n+1$ and calculate the generalized cost of the segment based on existing passenger flow. $t_{e}^{n}=t_{e}\left(x_{e}^{n-1}\right), \forall e \in E$ : the remaining capacity of each segment returns to the initial value, $z_{e}^{n}=h_{e}, e \in E_{1}$ : each $\mathrm{OD}$ demand returns to the initial value.

Step 3. Passenger flow assignment 
Step 3.1. Randomly select $\alpha_{o}^{n}$ from the distribution function of value of time for each station as the value of time of all passengers who depart from this station.

Step 3.2. Randomly select OD pair $w$, list all its service paths, calculate their generalized costs, and put the generalized costs of all paths into the set $R_{w}$ from small to large.

Step 3.3. Select the path $r_{w}^{1}$ with the lowest generalized cost from $R_{w}$, check the remaining capacity of the segments that it includes $z_{e}^{n}\left(\delta_{e r^{w}}=1\right)$, and take the minimum remaining capacity as its available capacity $z^{n}\left(r_{w}^{1}\right)$; allocate OD demand of $\min \left\{z^{n}\left(r_{w}^{1}\right), g_{w}\right\}$ into the path $r_{w}^{1}$ and update the remaining capacity of corresponding segment $z_{e}^{n}=z_{e}^{n}-$ $\min \left\{z^{n}\left(r_{w}^{1}\right), g_{w}\right\}$ and OD demand $g_{w}=g_{w}-\min \left\{z^{n}\left(r_{w}^{1}\right)\right.$, $\left.g_{w}\right\}$, if $g_{w}=0$, go to Step 3.4; otherwise, select the secondary path $r_{w}^{2}$ and repeat Step 3.3.

Step 3.4. If some OD pairs are not distributed in the set of $W$, go back to Step 3.2; if all OD demands are distributed, then obtain the extra flow $y_{e}^{n}$ of each segment and move to Step 4.

Step 4. Upgrade the flow of the segment, and calculate the flow of each segment $x_{e}^{n}$ after $n$ times of iteration through MSA method, namely,

$$
x_{e}^{n}=x_{e}^{n-1}+\eta_{n}\left(y_{e}^{n}-x_{e}^{n-1}\right), \quad 0 \leq \eta_{n} \leq 1 .
$$

Step 5. Convergence judgment. If certain times of iteration are completed or the requirements of convergence indicator $\Psi=\max \left(\sum_{k=1}^{n} \eta_{k} x_{e}^{k} / \sum_{k=1}^{n} \eta_{k}-\sum_{k=1}^{n-1} \eta_{k} x_{e}^{k} / \sum_{k=1}^{n-1} \eta_{k}\right)$ are met, stop calculating and $x_{e}^{n}$ is the final result of assignment; otherwise, go back to Step 2.

\section{Case Study}

Python programming is used to construct an algorithm of the proposed passenger flow assignment model, and the actual data of Beijing-Shanghai high-speed railway are imported into the algorithm to verify the validity of the algorithm. The physical network in this case consists of 23 stations on Beijing-Shanghai high-speed railway and segments between stations, and the training operation network includes 44 G-headed trains running downward from Beijing to Shanghai and 257 passenger flow ODs. It is inconvenient for passengers to make too many transfers; therefore, no more than one transfer is restricted before the service path is generated.

Assume that the probability density function of passengers' value of time obeys the rules of logarithmic normal distribution [12], namely,

$$
f(\alpha)=\frac{1}{\beta \sqrt{2 \pi}} \alpha^{-1} \exp \left[-\frac{1}{2}\left(\frac{\ln \alpha-\lambda}{\beta}\right)^{2}\right], \quad 0<\alpha<\infty, \beta>0 .
$$

In the equation, $\lambda$ and $\beta$ are the average value and standard deviation of $\ln \alpha$, respectively. The relations between their values, the average value $\alpha$, and standard deviation $\sigma$ are defined as

$$
\begin{gathered}
\lambda=\ln \mu-\frac{1}{2} \beta^{2}, \\
\beta^{2}=\ln \left(1+\frac{\sigma^{2}}{\mu^{2}}\right) .
\end{gathered}
$$

Value of time can be expressed as people's hourly wage. According to the average annual incomes of urban residents released by China Statistical Yearbook, the average value of time of the passengers in seven places where BeijingShanghai high-speed railway runs through including Beijing, Tianjin, Hebei, Shandong, Anhui, Jiangsu, and Shanghai is RMB 34/h with a standard deviation of RMB $11 / \mathrm{h}$ based on 251 legal workdays per year. According to equation (11)-(13), the distribution of the value of time for passengers on Beijing-Shanghai high-speed railway can be obtained as Figure 4.

The calculation coefficient of the generalized cost of the path $\beta_{T}, \beta_{P}$, and $\beta_{d e}$ is set to be 1,1 , and 0.5 , respectively, and passengers' onboarding and off-boarding speed $Q$ is set to be 40 people/min. After a day is divided into five time slots, which are 7:00 to $10: 00,10: 00$ to $13: 00,13: 00$ to $16: 00,16: 00$ to $19: 00$, and after 19:00, the proportions of passengers within each time slot to the total number of passengers in the whole day can be obtained based on the statistics of passenger tickets in peak seasons of 2015 for Beijing-Shanghai high-speed railway. The data extracted from peak seasons allow such proportions to approximately reflect passengers' preferences on departure time, Hence, the values of parameters $F(1), F(2), F(3), F(4)$, and $F(5)$ are set to be $13 \%$, $25 \%, 28 \%, 23 \%$, and $11 \%$, respectively, and the updated parameter $\eta_{k}$ of the flow in segment as 0.5 , times of iteration as 100 , and convergence indicator as 10 . The current ticket fare of Beijing-Shanghai high-speed railway is taken as input data, and the demand in a certain day of peak seasons is selected as OD demand for assignment. The requirements of convergence indicator can be satisfied after 63 times of iteration. Figure 5 illustrates how convergence indicator changes, and Figure 6 shows the comparison between the average occupancy rate obtained from passenger flow assignment and actual statistics.

In Figure 6, grey bar represents the actual occupancy rate of the train obtained based on ticket data, and white bar expresses the occupancy rate obtained based on the results of passenger flow assignment. By comparison, it can be seen that both bars are well coincided, among which the errors of 28 trains are less than $10 \%$, accounting for $64 \%$ of the total number of experimental trains. Thus, it can be concluded that this method can be used to simulate passengers' options and predict the assignment of passenger flow between trains at a certain ticket fare. According to the contrast, the calculation results of eight trains deviate from the actual value by $30 \%$. After analysis, it is found that the arrival time at the destination station of all these eight trains is later than 20:00, meaning that they are nonpreferred trains in terms of arrival time, which is not reflected in the proposed model. It is 


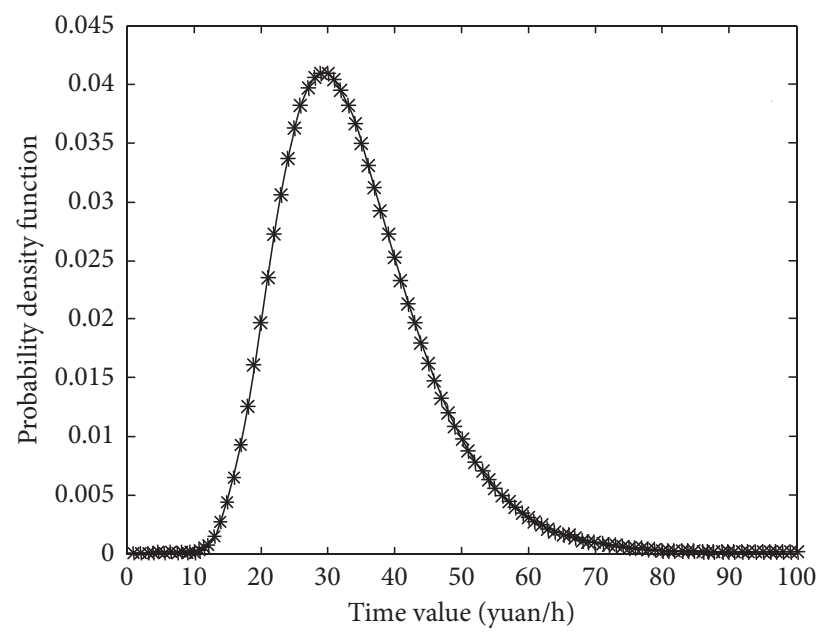

* Mean value 34 yuan $/ \mathrm{h}$

Standard deviation 11 yuan $/ \mathrm{h}$

FIgURE 4: Distribution of passengers' value of time.

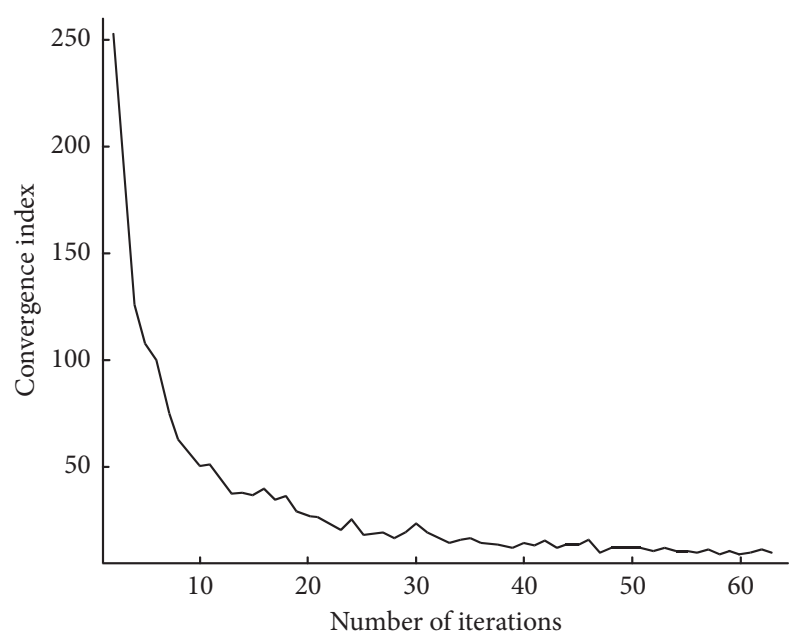

FIGURE 5: Changing curve of convergence indicator.

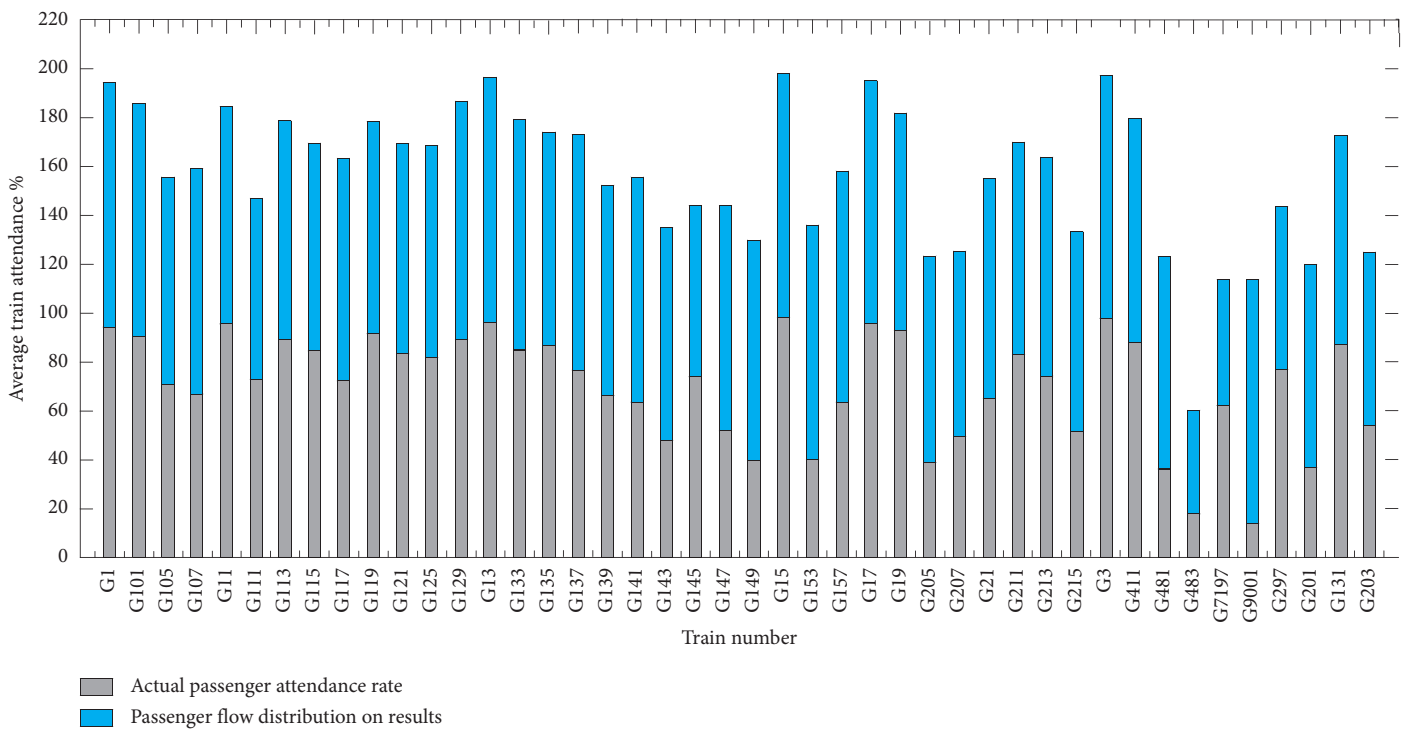

FIgURE 6: Comparison between actual occupancy rate and calculation result. 


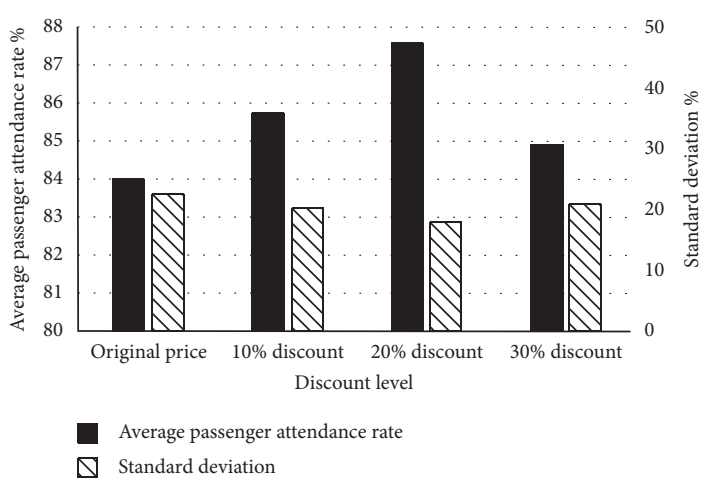

(a)

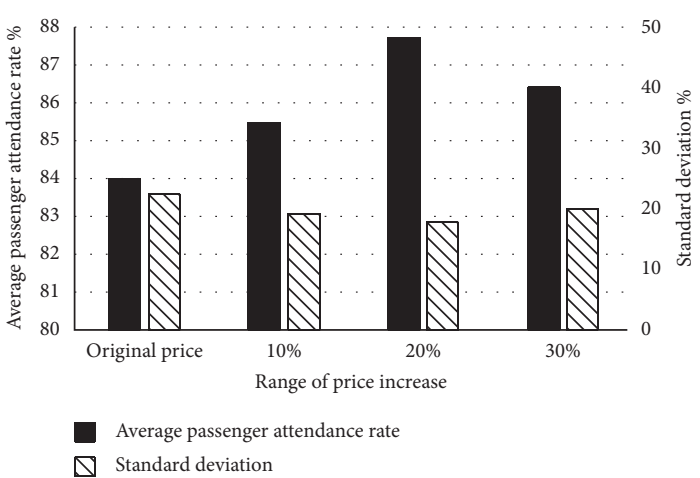

(b)

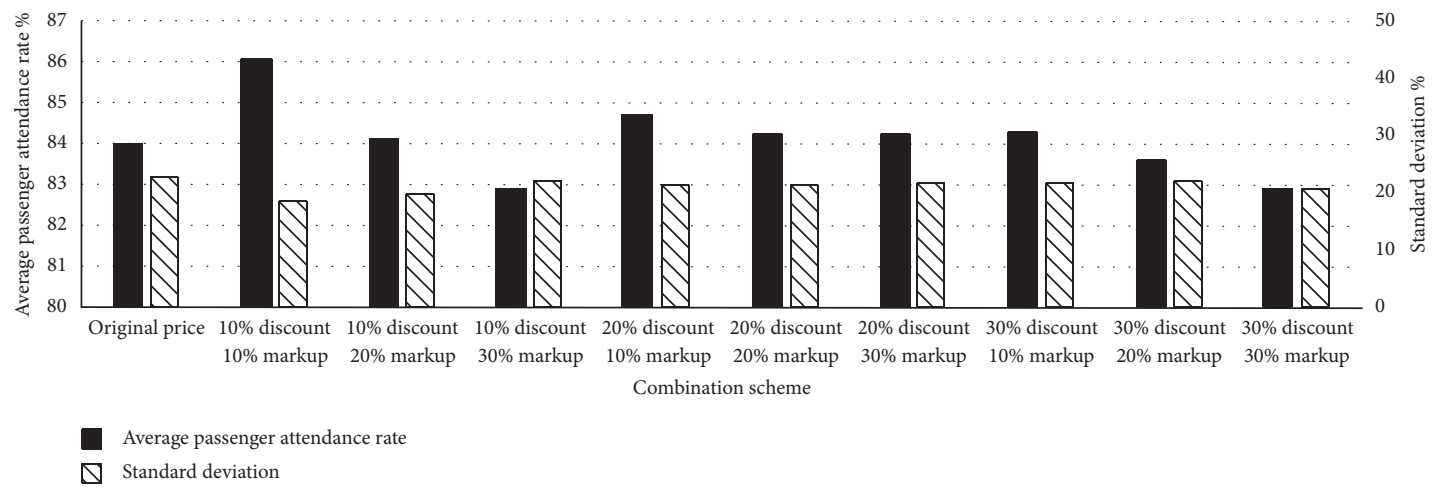

(c)

Figure 7: Occupancy rate and standard deviation under different fare schemes.

recommended to consider passengers' preferences for arrival time while analyzing passengers' options so as to make the model of passenger flow assignment more practical.

The strategy of optimizing high-speed train ticket fare is discussed based on the proposed passenger flow assignment method. Five trains with lower actual occupancy rate, namely, G483, G7197, G203, G207, and G297, and five trains with higher actual occupancy rate, namely, G1, G13, G15, G3, and G17, are taken as the objects of fare adjustment. Three kinds of strategies of adjusting ticket fare are adopted, which are to only reduce fare for trains with lower occupancy rate, to only increase fare for trains with higher occupancy rate, and simultaneously reduce or increase fare for these trains. The occupancy rate is summarized based on the passenger flow assignment results under different fare schemes and then compared with that at the original ticket fare. The details are shown in Figure 7.

It can be seen from the comparison of occupancy rate between fare reduction and original fare as indicated in Figure 7(a) that the occupancy rate after fare is reduced is higher than that at the original fare. As discount increases, occupancy rate increases firstly but later decreases, while its corresponding standard deviation witnesses an opposition trend, indicating that there is a threshold value of discounted fares in the strategy of only reducing fare to make passenger assignment the most balanced. It can be seen that $20 \%$ discount is the optimal multiclass fare scheme in this case. Similarly, in Figure 7(b) where fare is only increased, as the markup increases, the occupancy rate also increases firstly and later decreases. In this case, 20\% markup for preferred trains is the optimal multiclass fare scheme. According to Figure 7(c) where integrated strategy is implemented and compared, when the discount level is fixed, the greater the markup level, the worse balanced the passenger flow distribution, which indicates that the difference between the fares of markup trains and markdown trains should not be too large in the combination strategy. The optimal multiclass fare scheme in the integrated strategy in the given case is $10 \%$ for five subselected trains and 10\% markup for five preferred trains.

\section{Conclusions}

This paper studies the method of passenger flow assignment for high-speed trains, constructs a three-dimensional timespace service network as passenger flow assignment network, and designs the generalized cost to demonstrate passengers' travel cost during the whole journey. Moreover, passengers' value of time is described as continuously distributed random variable, and they are allowed to make their preferred option between time and expense. A bi-criteria equilibrium passenger flow assignment model is built and an improved Monte-Carlo random simulation algorithm is designed for passenger flow assignment. The actual data about passenger fares for Beijing-Shanghai high-speed railway are used as input data for passenger flow assignment. The results show that time-expense bi-criteria passenger flow assignment method can better predict the assignment at a 
certain fare. Lastly, the strategies of optimizing ticket fare are primarily discussed based on the proposed passenger flow assignment method.

\section{Data Availability}

The data used to support the findings of passengers' time value have been deposited in China Statistical Yearbook (http://www.stats.gov.cn/tjsj/ndsj/2015/indexch.htm). However, the ticket sale data used to support the findings of passenger flow allocation were supplied by Jinghu High-speed Railway Co. LTD under license and so cannot be made freely available.

\section{Conflicts of Interest}

The authors declare that they have no conflicts of interest.

\section{Acknowledgments}

This research was supported by the major scientific research project of China Academy of Railway Sciences Corporation Limited (2019YJ106).

\section{References}

[1] D. E. Boyce, L. J. Leblanc, and K. S. Chon, "Network equilibrium models of urban location and travel choices: a retrospective survey*," Journal of Regional Science, vol. 28, no. 2, pp. 159-183, 1988.

[2] M. Patriksson, The Traffic Assignment Problem: Models and Methods, VSP BV, Utrecht, The Netherlands, 1994.

[3] D. Lian-bo, F. Shi, and Q. Jin, "Passenger assignment on the base of passenger train plan," in Proceedings of the Sustainable Development of China's Transportation: Doctoral Forum of China (Traffic and Transportation Engineering), China Railway Publishing House, Beijing, China, 2005.

[4] T. Lu, "Research on passenger flow assignment method based on complex train service network," Journal of the China Railway Society, vol. 34, no. 10, pp. 7-15, 2012.

[5] N. Lei, "Research on passenger flow assignment based on passenger train schedule," Journal of Transportation Systems Engineering and Information Technology, vol. 11, no. 3, pp. 87-92, 2011.

[6] F. Shi and D. Lian-bo, "Optimal design of passenger transfer network," Journal of Railway Science and Engineering, vol. 1, no. 1, pp. 78-82, 2004.

[7] E. Cascetta, Transportation Systems Analysis: Models and Applications, Springer Science \& Business Media, Berlin, Germany, 2009.

[8] G. Xu, H. Yang, W. Liu, and F. Shi, "Itinerary choice and advance ticket booking for high-speed-railway network services," Transportation Research Part C: Emerging Technologies, vol. 95, pp. 82-104, 2018.

[9] S. C. Dafermos, "The traffic assignment problem for multiclass-user transportation networks," Transportation Science, vol. 6 , no. 1, pp. 73-87, 1972.

[10] F. Leurent, "Cost versus time equilibrium over a network," European Journal of Operational Research, vol. 71, no. 2, pp. 205-221, 1993.

[11] H. Yang and H.-J. Huang, "The multi-class, multi-criteria traffic network equilibrium and systems optimum problem,"
Transportation Research Part B: Methodological, vol. 38, no. 1, pp. 1-15, 2004.

[12] H. Yang, H. Y. Kong, and Q. Meng, "Value-of-time distributions and competitive bus services," Transportation Research Part E: Logistics and Transportation Review, vol. 37, no. 6, pp. 411-424, 2001. 